\title{
WAVELET DECOMPOSITION IN THE EARTH'S GRAVITY FIELD INVESTIGATION
}

\author{
Janusz BOGUSZ $^{1) *}$, Anna KLOS ${ }^{1)}$ and Wieslaw KOSEK ${ }^{2), 3)}$ \\ 1) Centre of Applied Geomatics, Military University of Technology, 00-908 Warsaw, Kaliskiego St. 2, Poland \\ ${ }^{2)}$ University of Agriculture, Environmental Engineering and Land Surveying, 30-059 Kraków, \\ Mickiewicza Av. 24/28, Poland \\ 3) Polish Academy of Sciences, Space Research Centre, 00-716 Warsaw, Bartycka St. 18A, Poland \\ *Corresponding author's e-mail: jbogusz@wat.edu.pl
}

(Received November 2012, accepted January 2013)

\begin{abstract}
This paper presents the results of the application of wavelet decomposition to processing data from the GGP sites (The Global Geodynamics Project). The GGP is an international project within which the Earth's gravity field changes are recorded with high accuracy at a number of stations worldwide using superconducting gravimeters. Data with a 5-second sampling interval from Wettzell and Bad Homburg were used for the research. The wavelet transform enables the investigation of the temporal changes of the oscillation amplitudes or the decomposition of the time series for the analysis of the required frequencies. The wavelet decomposition was performed using the regular orthogonal symmetric Meyer wavelet. The research concerned data from an earthquake period recorded at various locations and a quiet period when the gravimeters worked without any disturbances. The decomposition was followed by the Fast Fourier Transform for signal frequency components and then by correlation analyses of corresponding frequency components (for periods from 10 to 60000 seconds) for all sensor combinations, for the quiet and the earthquake periods separately. Frequency components defining long term changes for all sensor combinations, as well as combinations between two sensors at the same site for the quiet days are characterised by high correlation coefficients. For the time of the earthquake, the Wettzell site data proved strong correlation for all frequency components, while the Bad Homburg site data showed an unexpected decrease of correlation for the majority of frequency components. The authors also showed that wavelet decomposition can be a good method of data interpolation, especially from the time of earthquakes. Moreover, it is a very useful tool for filtering the data and removing the noises.
\end{abstract}

KEYWORDS: GGP, wavelet decomposition, Earth's gravity field changes

\section{INTRODUCTION}

Gravimetry is a science dealing with the measurements of the Earth's gravity, i.e. the intensity of the Earth's gravity field. Gravimetry includes measurements of the temporal changes of the Earth's gravity field. The value of gravity at a location depends, among others, on the tidal forces and internal structure of the Earth. Changes in the Earth's gravity field are recorded e.g. within the confines of the GGP (The Global Geodynamics Project) that combines and makes available observations from numerous stations all over the world. Changes in the Earth's gravity field are recorded at one, two, five and sixty second intervals and are freely available through the Internet. During the quiet time, the gravimeters data, after removal of the measurement systems' noise, enable the detection of the real changes in the Earth's gravity field. In non-quiet periods, numerous anomalies are caused by, among others, earthquakes, i.e. the Earth's crust vibrations generated by the propagation of seismic waves. This anomalous behaviour is analysed to determine its impact on the gravimeter performance.

Wavelet transform is widely applied for temporal and frequency analyses of signals as well as a band- pass filter. The latter case is called wavelet decomposition. It is used nowadays in as many fields of science as the filtering of data or decomposition of time series into different frequency components as needed. Kosek and Popinski (1996) used wavelet decomposition based on Meyer (Meyer, 1990; Popinski and Kosek, 1995) and Shannon (Benedetto and Frazier, 1994) wavelet functions for adaptive forecasting of pole coordinates time series in different frequency bands. Gurley and Kareem (1999) introduced the wavelet transform to the analysis of earthquake, wind and wave data. They decomposed the mentioned time series with the use of discrete or continuous wavelet transform. They presented the examples of spectral and co-spectral analyses, timescale decomposition, that was made to identify the transient events, monitoring via scalograms, the clearing of noisy signals and non-stationary signal simulations. Lundstedt et al. (2006) presented the results of wavelet-based analysis (including the multiresolution analysis) of the long-term solar activity in the form of scalograms, skeletons, ampligrams and the time scale spectra. The above methods have helped the authors to show the similarities and differences between the used data sets. Moreover, they have 
found many different periodicities and have presented their changes with time. Lark and Webster (1999) used wavelets to analyse the soil variation in two regions (in Australia and England). They showed the power and merits of multi-resolution analysis and the wavelet transform. Hassler et al. (2011) showed the variations in soil beneath a tropical rain forest with the use of the multi-resolution analysis. The authors also calculated the wavelet packet variance and the wavelet correlation coefficients. Chamoli et al. (2010) analysed the tsunami-generating and non-tsunamigenerating earthquakes recorded at different stations to identify the tsunami-genesis of an earthquake. The authors examined the frequency of the seismogram components in the time domain using wavelet transform. The threshold of frequencies for tsunami warnings was estimated. They proved that only a few minutes of recording are enough for a quick estimation of possible tsunami regions. Sang (2012) decomposed the two hydrologic time series: the simulated with the Monte-Carlo method and the real energy function. By comparing the above data sets, the author proposed a few ways of using discrete wavelet transform for analysing time series. His article is a type of practical guide in how to use the wavelets.

$\mathrm{Hu}$ et al. (2007) used the band-pass filtering with Daubechies wavelet for eliminating noise in superconducting gravimeters at four European stations. These helped the authors to estimate the gravimetric factor of the Chandler wobble. $\mathrm{Hu}$ et al. (2005) proposed a new method to process gravity and pressure signals with the use of Daubechies wavelet. The method was tested on observations from the Wuhan station with a superconducting gravimeter. It was proved that the atmospheric pressure effects have their influence on gravity variations. Hu et al. (2006) processed the superconducting gravimeter data containing the Sumatra-Andaman earthquake with a wavelet method to remove the local atmospheric pressure fluctuations from gravity changes. This research showed the improvement of evaluation of the coupled mode ${ }_{1} \mathrm{~T}_{2}$ and ${ }_{1} \mathrm{~T}_{3}$ and the splitting singlets of mode ${ }_{2} \mathrm{~S}_{1}$. The correction of gravity observations with band-pass filters can improve the signal-to-noise ratio of weak signals in the elaboration of long period normal mode seismology. Enescu et al. (2004) presented the results of the in-depth analysis of the multi-fractal and correlation properties of real and simulated time series of earthquakes. The real earthquake data came from the region of the epicentre of the Kobe earthquake in 1995. The simulated one, from the Vrancea region, Romania. The analysis of simulated time series revealed that for the immense range of scales the process does not show any correlations. The real earthquake data proved the different types of fractals depending on scales. At small scales the time series were multi-fractal and for the larger mono-fractal. To confirm the results the authors considered two "artificial" earthquake sequences. The multi-fractal analysis based on wavelets showed several distinct scaling domains. Lintao et al. (2000) proposed a new method for the determination of gravity tide parameters. With the use of three pairs of wavelets the tides were extracted from the gravity series by filtering. The research was conducted on the Wuhan superconducting gravimeter records. Liye and Hongsheng (2011) proved the predominance of the morphological wavelet filter algorithm on the classical wavelet filter algorithm. The noise was removed from the precise gravimeter signal and then it was reconstructed without noise. Xiaogang et al. (2006) used the wavelet-based filtering with narrow band-pass frequency on data from superconducting gravimeters after the SumatraAndaman earthquake. The authors cleared the observations of nearly all coupled toroidal modes with the frequencies below $1.5 \mathrm{mHz}$. What is more, two modes were revealed in the vertical component of free oscillations for the first time. Greco et al. (2007) proposed the processing of data with the use of multiresolution analysis to filter the noise from the gravity data. They collected the mentioned data on an active volcano with the aim to detect mass changes connected with magma processes. The evaluation of the traditional filtering methodology and the wavelet transform were also done.

The gravimeters record the sum of gravity changes, which not only consists of tidal gravity but also the gravity effects on global, regional and local scales, e.g. environmental influences, noise or instrumental effects. The main goals of this paper were the band-pass filtering of data, proving that the unwanted frequencies can be excluded from the signal as well as checking if there are any dependences between the gravity signals recorded at the same station (but using the various sensors) and the neighbouring stations at the time of earthquakes and the so called quiet days.

\section{DATA}

The observations from the GGP project stations were used for the research. The GGP is an international gravimetric project recording changes of the Earth's gravity field by means of superconducting gravimeters at stations located worldwide (Crossley and Hinderer, 2010; Crossley et al., 1999). These observations are widely used for determining the Chandler periods and for investigating the gravity effects resulting from changes in the atmospheric pressure or the groundwater level. Earthquakes are the anomalous phenomena, otherwise modern geodetic instruments record the full spectrum of the existing environmental signals. Examples of such sensors may be either gravity sensors (e.g. Krause et al., 2009; Kroner, 2008; Kroner et al., 2009) or GPS antennas (e.g. Bogusz et al., 2011). However, due to different instrumental properties (sensitivity), data from superconducting gravimeters are a much better source of information on changes in high frequencies (Banka 
and Crossley, 1999; Kroner and Weise, 2011) than GPS (Bogusz and Hefty, 2011; Bogusz and Kontny, 2011; Bogusz and Figurski, 2012).

The five seconds sampling data from the Wettzell and Bad Homburg stations (Fig. 1) were used for the described research. At these stations, the changes of the Earth's gravity field are recorded by means of dual sensor superconducting gravimeters with the upper and lower sensors. The superconducting gravimeters belong to the massspring ones. The difference is that they have no spring. It is replaced by the proof mass that is levitating in a magnetic field of superconducting coils. The gravity changes are recorded with a high precision. The main advantages of superconducting gravimeters are low (almost linear) drift, long term stability and high sensitivity on the level of $0.1 \mathrm{~nm} / \mathrm{s}^{2}$.

The analysed data concerned two periods: the quiet period when the gravimeters worked without any disturbances and the Sumatra-Andaman earthquake period.
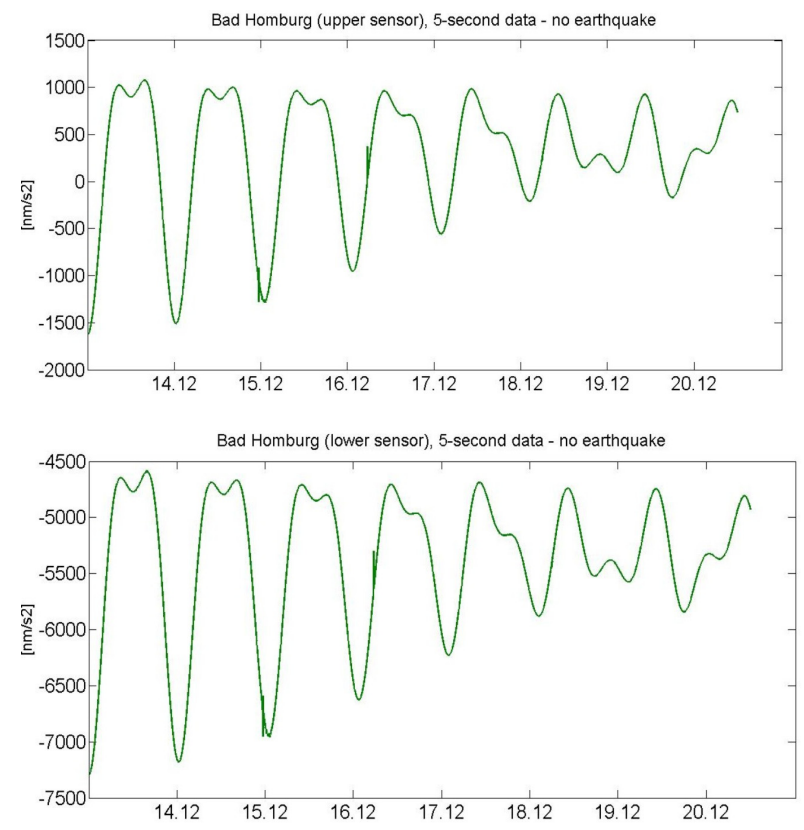

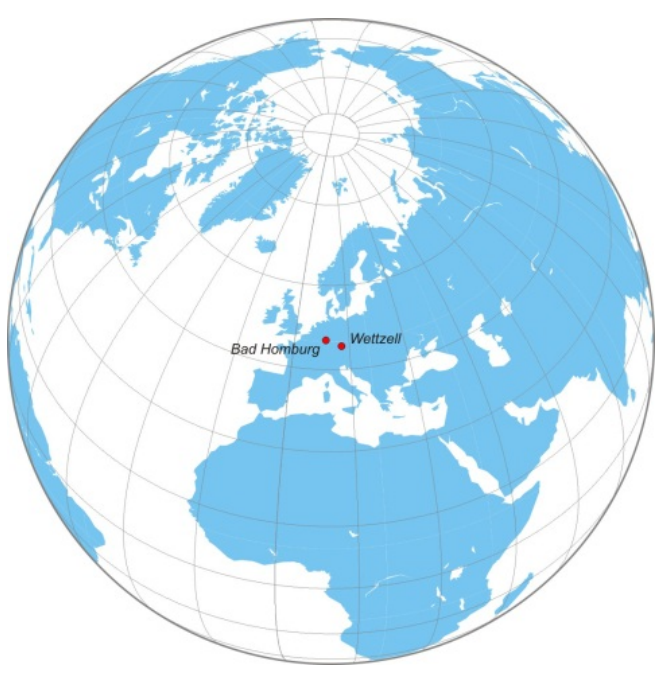

Fig. 1 Stations“ location.
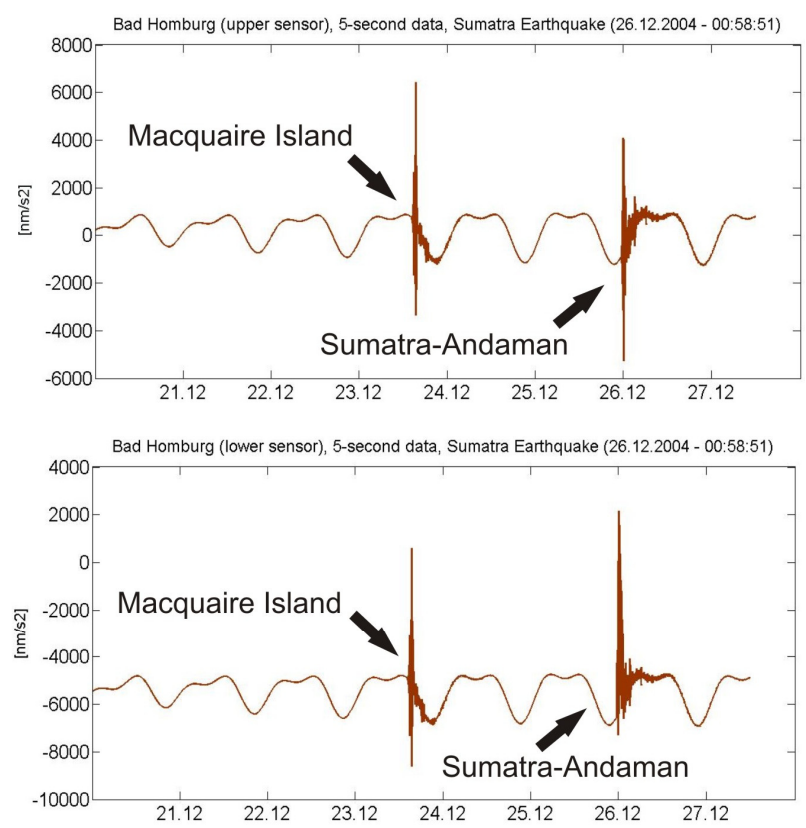

Fig. 2 Time series for the Bad Homburg station (at left: the quiet period; at right: the Sumatra-Andaman earthquake; top: upper sensor; bottom: lower sensor).

The former type of data (without disturbances) concerned the period from December 13 through to the 20,2004. The latter, including the time of the earthquakes, started on December 20 and ended on December 27, 2004 (Fig. 2 and Fig. 3).

The Sumatra-Andaman 9.1 (NEIC) earthquake took place at 00:58:53 UTC on December 26, 2004 with its epicentre at $3.50^{\circ} \mathrm{N}, 95.72^{\circ} \mathrm{E}$. It was preceded by another earthquake recorded at the northern part of the Macquaire Island (at 14:59:03 UTC with its epicentre at $50.15^{\circ} \mathrm{S}, 160.37^{0} \mathrm{E}$ and magnitude of 8.1(NEIC)).

\section{WAVELET DECOMPOSITION}

The wavelet decomposition method consists of decomposing the signal into a series of functions with decreasing frequencies in such a way that each level of decomposition includes a high frequency component and an approximation resulting from extracting the high frequency components from the signal (Fig. 4).

The wavelet decomposition method has an important feature, depending on the sort of the chosen wavelet - the sum of all the frequency components from the individual levels and the final approximation yields of the initial signal. 



Fig. 3 Time series for the Wettzell station (at left: the quiet period; at right: the Sumatra-Andaman earthquake; top: upper sensor; bottom: lower sensor).

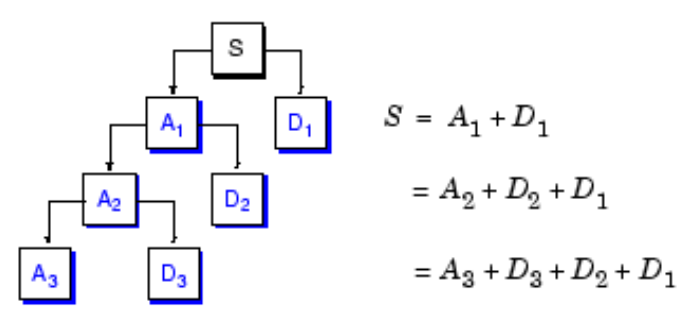

Fig. 4 Decomposing the signal into the individual levels (Missiti, 2000).

The frequency components of the signal for the certain scale $j$ are obtained by means of the decomposition process of the $x(t)$ series using the discrete wavelet transform.

For all time series of the length of $N=2^{p}(N-$ number of data, $p-$ number of frequency components), the scale (dilation) index $j=-p,-p+1, \ldots,-$ 1 and the translation (shift) index equal to $k=0,1, \ldots, 2^{j} N-1$, there is a set of discrete wavelet functions $w_{j k}(t)$ such that its Fourier transform is (Popinski, 1996):

$w_{j, k}(v)=2^{\frac{-j}{2}} \cdot e^{\frac{-i \cdot 2 \pi \cdot 2^{-j} \cdot v \cdot k}{N}} \cdot \hat{h}^{\frac{2^{-j} v}{N}}$,

$v=\frac{-N}{2}+1, \frac{-N}{2}+2, \frac{N}{2}$

$\bar{h}\left(\omega_{0}, \omega\right)=F T[h(t)]$ is the Fourier transform of $h(t)$, such that $\hat{h}\left(\omega_{0}, \omega\right)=A\left(\omega_{0}, \omega\right)$ for $\omega \in R$ and the central frequency of the spectral window $\omega_{0} \neq 0$ : $h(t)=\frac{1}{2 \pi} \int_{-\infty}^{+\infty} \widehat{h}\left(\omega_{0}, \omega\right) \exp (i t \omega) d \omega=\exp \left(i t \omega_{0}\right) h_{0}(t)$,

where:

$$
\begin{aligned}
h_{0}(t) & =\frac{1}{2 \pi} \int_{-\infty}^{+\infty} \widehat{h}(0, \omega) \exp (\text { it } \omega) d \omega= \\
& -\frac{1}{2 \pi} \int_{-2 \pi \lambda}^{2 \pi \lambda} A(0, \omega) \exp (\text { it } \omega) d \omega
\end{aligned}
$$

and $A$ is the boxcar transfer function of the filter that is real and non-zero in the surrounding of the $\omega_{0}$ frequency.

The vector's development coefficients are computed with the use of:

$S_{0,0}=\frac{1}{n} \sum_{t=0}^{N-1} x(t), S_{j, k}=\sum_{t=0}^{N-1} x(t) \cdot w_{j, k}(t)$

Taking all above into consideration, for each of the $j$ frequency component the Discrete Wavelet Transform makes it possible to obtain the decomposition of the finite time series using:

$s_{j}(t)=\sum_{k=0}^{2^{j}} S_{j k} w_{j k}(t)$ for $t=0,1, \ldots, N-1$

The process of the signal reconstruction from the $p$ frequency components is done by means of:

$\sum_{j=-p}^{-1} s_{j}(t)=x(t)$ 


\section{PROCESSING OF THE OBSERVATION DATA}

The gravimetric time series were decomposed by means of a symmetric orthogonal Meyer wavelet. This selection was determined by the fact that this wavelet allows the reconstruction of the input signal by summing the individual frequency components and the final approximation (Popinski, 1996). The compatibility of the original signal and its reconstruction was proved at the level of $10^{-4} \mathrm{~nm} / \mathrm{s}^{2}$ which is three times better than the sensitivity of the gravimeter. As a result of the decomposition process of the time series, diagrams were obtained for each time series at which each of the components represents a different frequency - the higher the number of the component, the lower the frequency is (Fig. 5 and Fig. 6). The number of the decomposition levels depends on the length of the time series. In each of the decomposition diagrams $s$ is the input signal, $a_{12}$ is the final approximation, and $d_{1}-d_{12}$ are the individual frequency components of the signal (Table 1). The oscillations at the starts and the ends of the series of the frequency components are an artificial effect due to cropping the signal by the weighting window of the wavelet transform.

All analyses were done using Matlab ${ }^{\circledR}$ software, licence No. 350334.

\section{THE FFT ANALYSIS}

The Fast Fourier Transform (FFT) is an integral transform from the time domain into the frequency domain decomposing the given temporal series into a sum of periodic functions, in such a way that the convolution of them yields the initial function (Singleton, 1969):

$$
g(f)=\int_{-\infty}^{+\infty} g(t) \cdot e^{-2 \pi \cdot t \cdot f} \cdot d t
$$

where $i$ is the imaginary unit, $t$ is time in seconds, and $f$ is frequency in hertz.

Application of the Fast Fourier Transform yields information concerning the power of the oscillation as a function of the period.

The Fast Fourier Transform was performed for each of the above described frequency components. As a result of this transform, oscillations in frequency ranges defined by the individual levels of the wavelet decomposition were determined.

Examples of the transform of the frequency components 1 through 3 for the Bad Homburg station (lower sensor) during the Sumatra-Andaman earthquake are presented in Figure 7.

Examples of the transform waveforms of the frequency components for the Bad Homburg station lower sensor during the earthquake are presented in Figure 8 . These are the three highest levels of the decomposition, long-period effects were observed at the transforms of the frequency components. Figure 9 presents a comparison of the transforms of the frequency components 1 through 3 for the $\mathrm{Bad}$ Homburg station between the lower (at left) and upper
Table 1 Frequency ranges of the individual decomposition levels.

\begin{tabular}{cc}
\hline $\begin{array}{c}\text { Frequency component } \\
\text { number }\end{array}$ & $\begin{array}{c}\text { Frequency range } \\
\text { (presented as period in } \\
\text { seconds) }\end{array}$ \\
\hline 1 & $<10 ; 28>$ \\
2 & $<20 ; 56>$ \\
3 & $<32 ; 112>$ \\
4 & $<64 ; 224>$ \\
5 & $<128 ; 448>$ \\
6 & $<256 ; 896>$ \\
7 & $<512 ; 1792>$ \\
8 & $<1024 ; 3584>$ \\
9 & $<2048 ; 7168>$ \\
10 & $<4096 ; 14336>$ \\
11 & $<8192 ; 28672>$ \\
12 & $<16384 ; 57076>$ \\
\hline
\end{tabular}

(at right) sensors. The diagrams of the transforms show that the amplitude values for the corresponding frequency components are at a similar level. The same relation was observed for the quiet period, which is rather not surprising.

\section{CROSS-CORRELATION ANALYSIS}

The cross-correlations between corresponding frequency components for all combinations of sensors, separately for the quiet and the earthquake periods, were calculated according to the following formula:

$r_{X Y}=\frac{\operatorname{cov}(X, Y)}{\sigma_{X} \sigma_{Y}}=\frac{\frac{1}{n-1} \sum_{i=1}^{n}\left(x_{i}-\bar{x}\right) \cdot\left(y_{i}-\bar{y}\right)}{\sqrt{\frac{\sum_{i=1}^{n}\left(x_{i}-\bar{x}\right)^{2}}{n-1}} \cdot \sqrt{\frac{\sum_{i=1}^{n}\left(y_{i}-\bar{y}\right)^{2}}{n-1}}}$

where $\operatorname{cov}(X, Y)$ is the cross-covariance, while $\sigma_{X}$ and $\sigma_{Y}$ are standard deviations for the $X$ and $Y$ series, respectively.

Cross-correlations between the corresponding frequency components for combinations of sensors from various stations are presented in Figure 10, for the quiet period at left and the Sumatra-Andaman earthquake period at right.

High values of the cross-correlation coefficients are characteristic for both periods for frequency components 10 through 12 , i.e. those defining longperiod changes. This means that the gravimeters respond similarly to long-period changes, and earthquakes have no significant impact on their lowfrequency readings. Another result would be strange for the gravimeters both for the time of quiet and the earthquake. High values of the correlation coefficients are also characteristic for frequency components 3 and 4 for the quiet period. An unexpected decrease of the correlation value is observed for frequency 


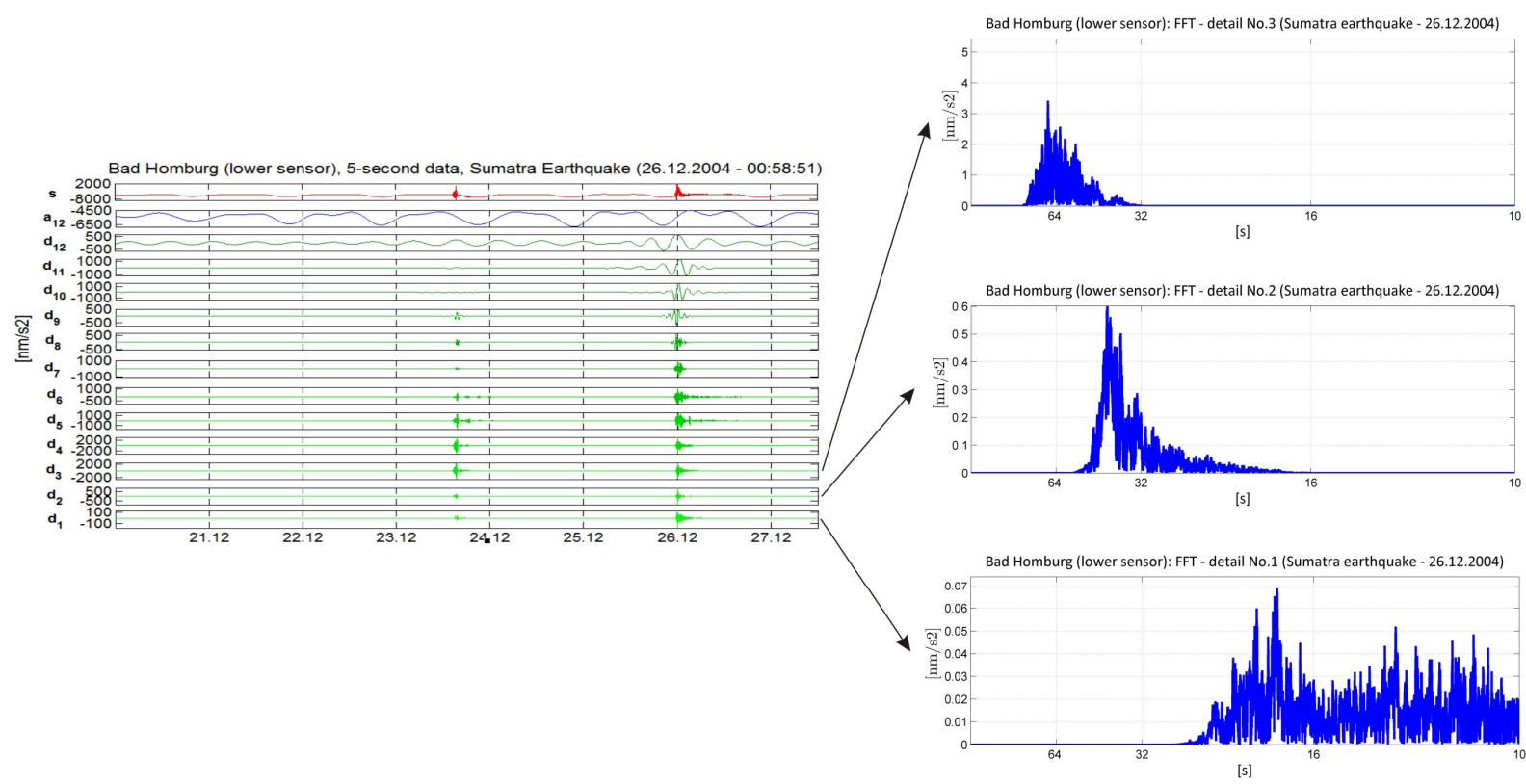

Fig. 7 The modules of the Fourier transform of the high frequency components for the Bad Homburg station (lower sensor) during the earthquake (top to bottom - frequency components 3, 2 and 1).

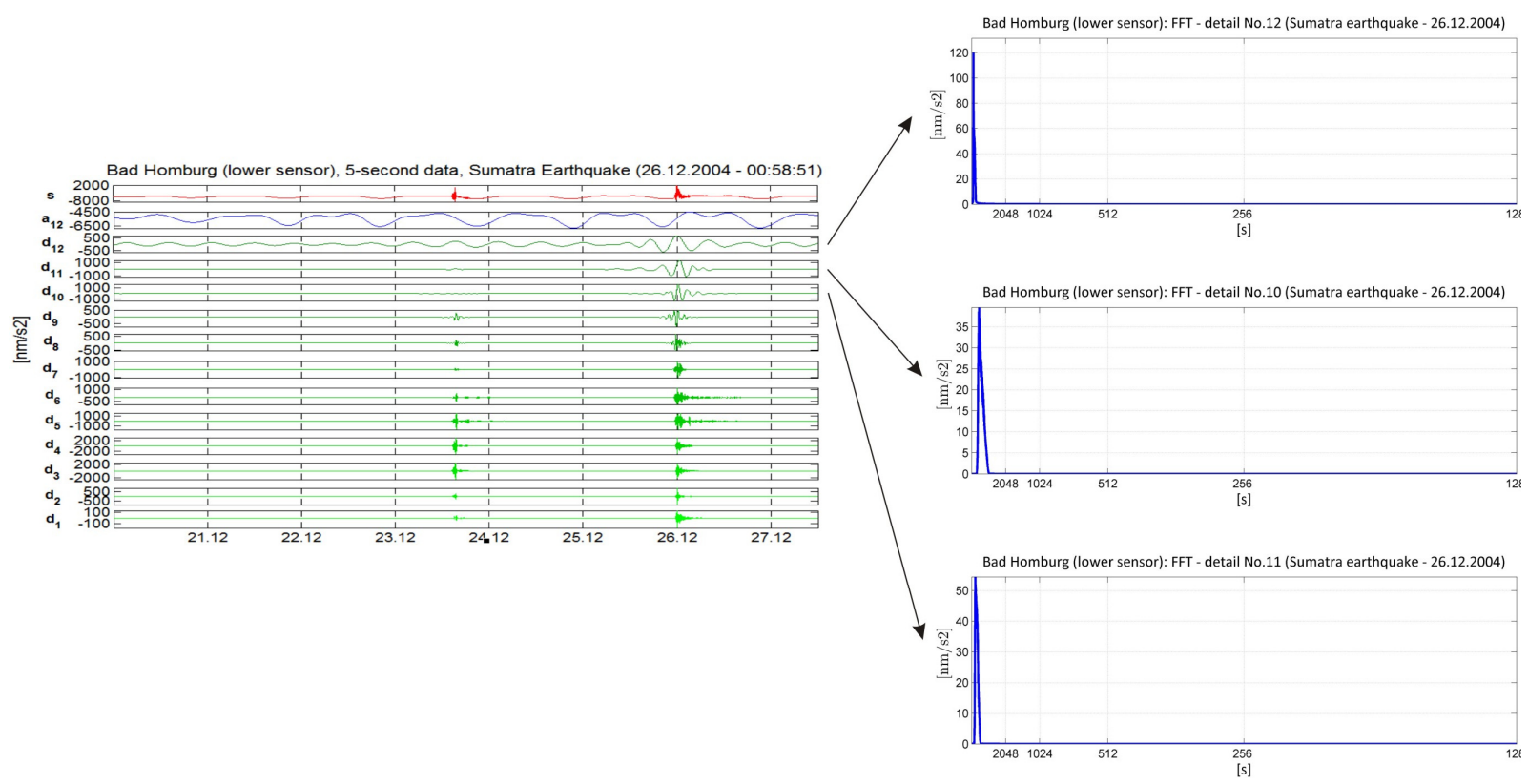

Fig. 8 The modules of the Fourier transform of the low frequency components for the Bad Homburg station (lower sensor) during the earthquake (top to bottom -frequency components 12, 11 and 10).

components 1 through 2 and 5 through 9 during the quiet period.

Frequency components 1 through 8 from the earthquake period are poorly correlated - the responses of the individual gravimeters during earthquakes are completely different depending on the frequency and amplitude of the seismic waves arriving at the station.

Cross-correlations between the corresponding frequency components for sensors from the same station are presented in Figure 11, for the quiet period at left and the Sumatra-Andaman earthquake period at right (the Wettzell station at the top, the Bad Homburg station at the bottom).

The two sensors at the same station for the quiet period have high values of the correlation coefficients - both sensors recorded the same changes in the Earth's gravity field. An unexpected decrease of correlation for the quiet period both for the Bad Homburg and the Wettzell stations appeared in the $6^{\text {th }}$ 

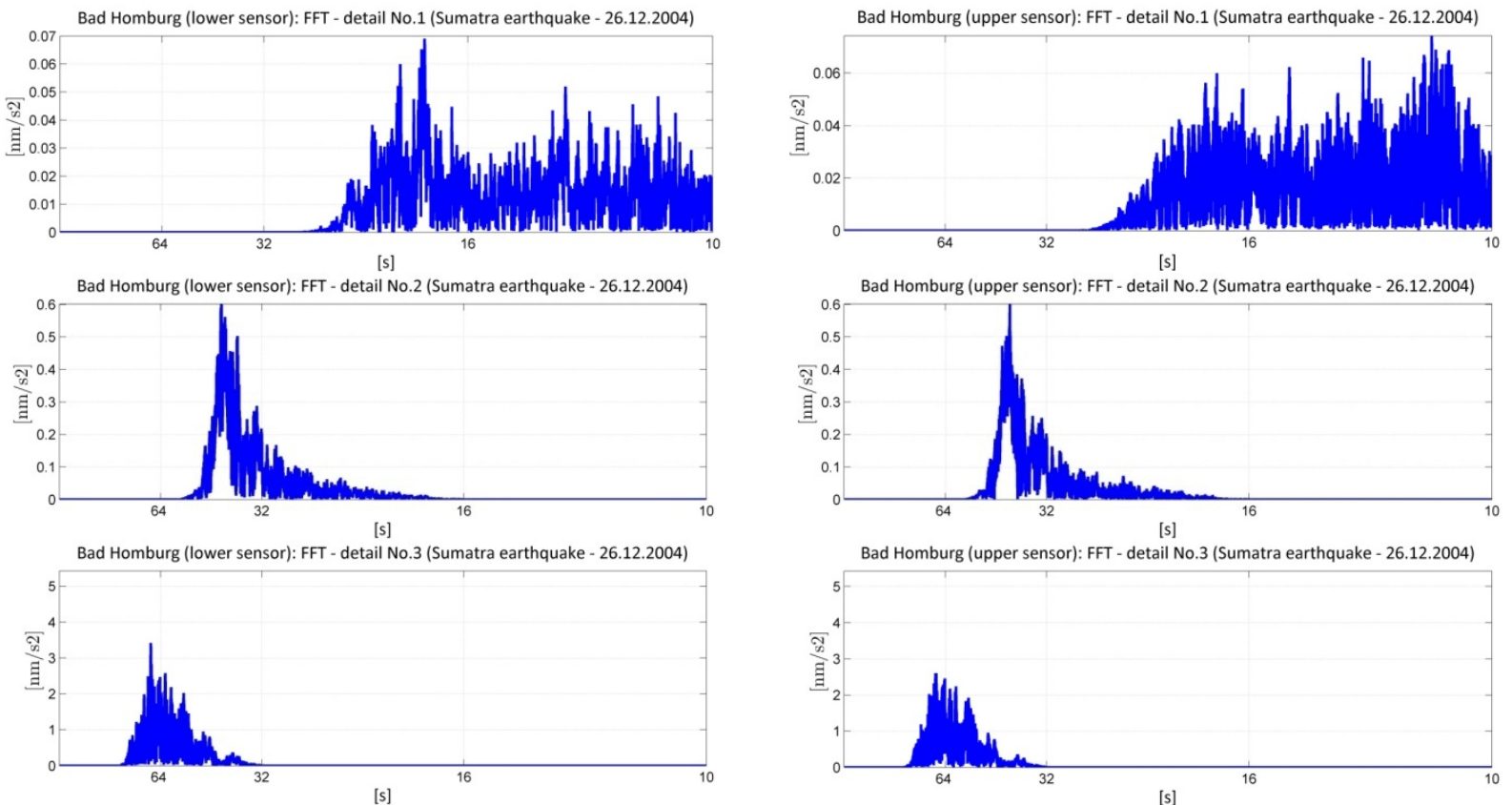

Fig. 9 The modules of the Fourier transform of the frequency components 1 through 3 (top to bottom) for the Bad Homburg station during the earthquake, at left - lower sensor, at right - upper sensor.
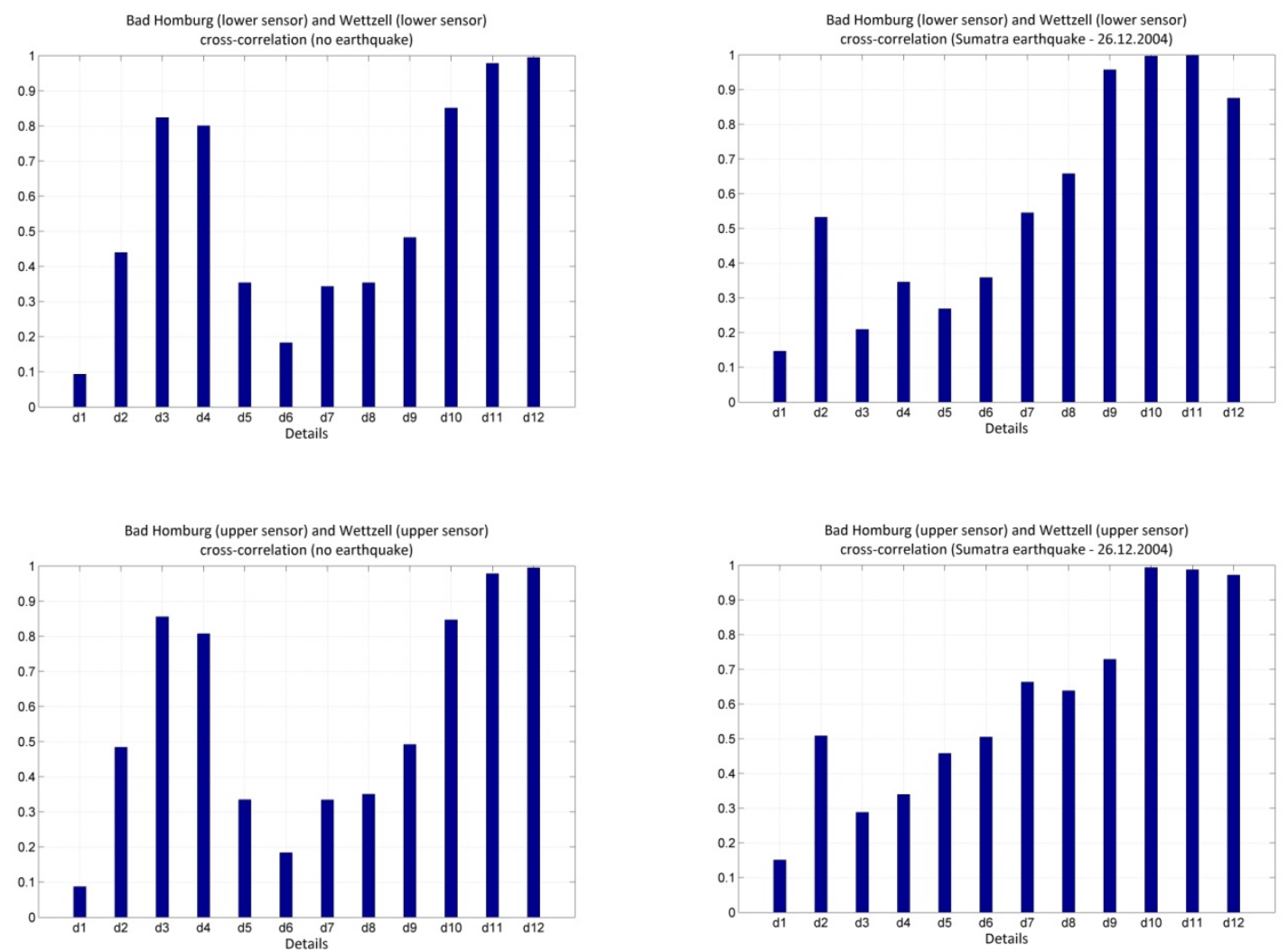

Fig. 10 Cross-correlations between corresponding frequency components for combinations of different stations. 

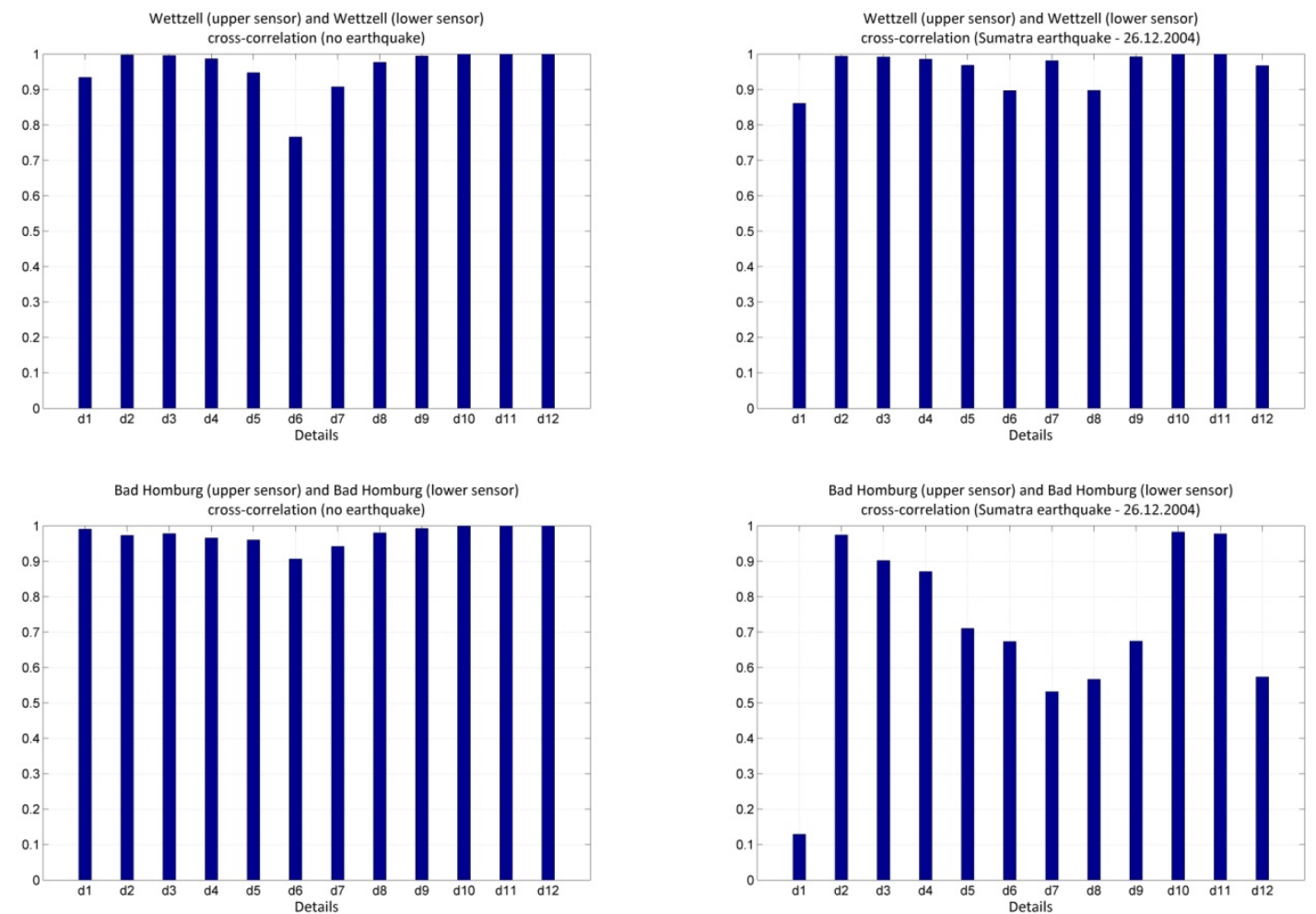

Fig. 11 Correlation coefficients between corresponding frequency components sensors from the same station.

frequency component (in the frequency range from 0.00112 to $0.00391 \mathrm{~Hz}$ ). During the earthquake the Wettzell station has high values of the correlation coefficients in all frequency components, while for the Bad Homburg station an unexpected decrease of correlation is observed for most of the frequency components.

\section{DISCUSSION}

1. The wavelet decomposition method presented in the described research enables one to obtain information concerning the response of gravimeters depending on the frequency and amplitude of disturbances as well as to compare two sensors recording the changes of the Earth's gravity field independently for each station.

2. The values of long-period changes are at similar levels during the earthquake and the quiet period in contrast to the high frequency components which values range changes unexpectedly.

3. The wavelet decomposition may be considered as a good method of data interpolation for earthquake periods (Fig. 12).

4. The wavelet decomposition enables the filtration of data in such a way that frequency components (i.e. for the scale index $j$ from 1 to 10 , which do not correspond to the tidal dynamic frequencies) excited by earthquakes are not taken into account in the signal reconstruction formula. In this way, the noisy data (earthquake) can be efficiently reduced from the original data set (comp.
Fig. 12). The using of the wavelet decomposition method as a band-pass filter has a very wide range of applications. It can be widely used for any kind of data to reduce the influence of undesired effects with known frequencies, as it was done for example in Bessissi et al. (2009) for DORIS data. It can also be applied for other geodetic time series (not only for gravity measurements, but the GPS time series as well) with any other sampling interval, dependent only on the desired frequencies that are to be obtained.

5. Signal approximation at the highest level of the decomposition after removing tidal effects may be a good representation of the instrument drift.

6. No characteristic frequencies for the time of the earthquake were found. The modules of the Fourier transform behave in an unpredictable way.

7. The cross-correlation between corresponding frequency components showed similar coefficients for all the combinations of sensors for the quiet time. The period ranges from 32 up to 224 seconds (components $2^{\text {nd }}$ and $3^{\text {rd }}$ ) and from 4096 up to 57076 seconds (components 10-12) are highly correlated. Further research concerning the cause of the high cross-correlation in the first range is needed. The second range of high crosscorrelation values appeared due to the removal of high frequency components (i.e. noise and other effects) and proved the same recording of changes in the mean of low-frequency tidal 


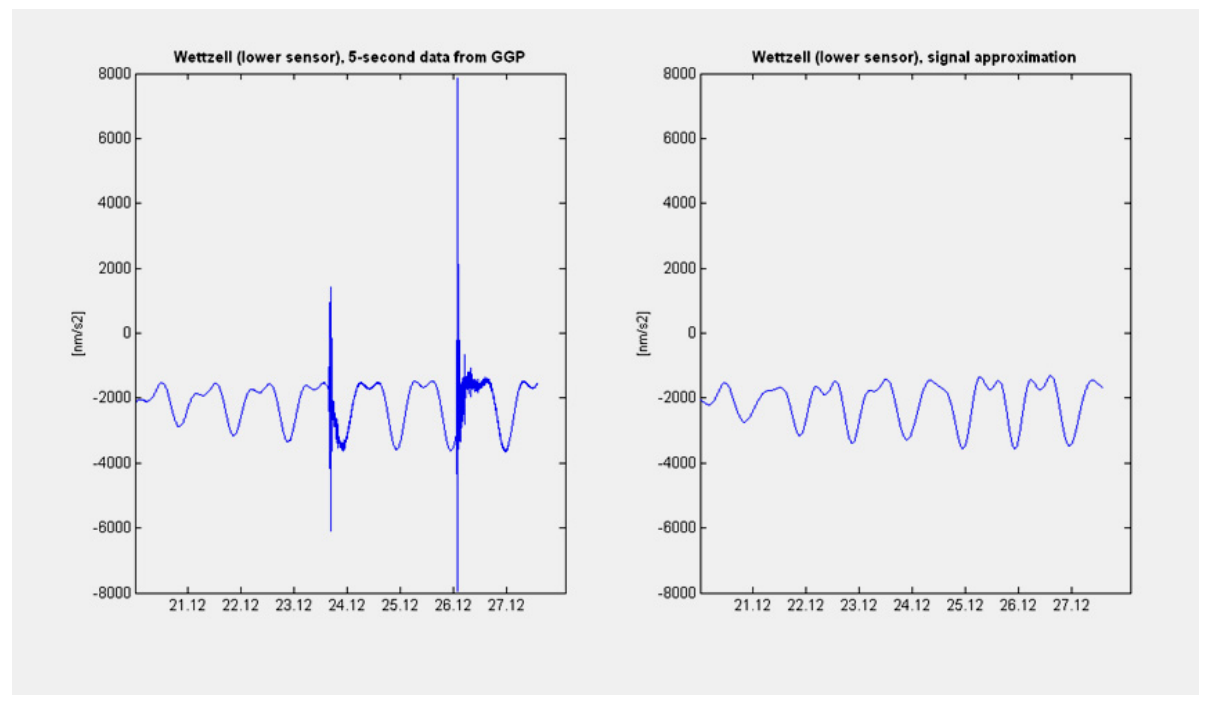

Fig. 12 The time series from the Wettzell station and its final approximation after removing the high frequency components.

effects. The cross-correlations from the period of the Sumatra-Andaman earthquake did not show any spatial dependence (the dependence between two described stations) in amplitudes of oscillations. At the beginning of the research we expected to obtain similar frequencies from the time of the earthquake (due to the very close distance between the two considered stations in comparison to the hypocenter). The similar range of high cross-correlation values can be noticed in the low frequency component at the time of the earthquake. It can be caused, as before, by removing the high frequency noise resulting from earthquake occurrence and leaving only the denoised, long-period changes. It is a good example of using wavelet decomposition as a band-pass filter.

8. The correlation coefficients from the quiet time between sensors from the same station are characterised by high values. It proves the same sensitivity of sensors and high agreement in recording the same effects. Despite the fact that both sensors recorded the same gravity changes we have found an unexpected decrease in coefficients appearing in the period of 256 up to 896 seconds ( $6^{\text {th }}$ component) for each station. The upper and lower sensors did not react in the same way during this period, which could be caused by some instrumental properties. The good agreement of the sensors' work is expected in any other period.

\section{ACKNOWLEDGMENTS}

The GGP service is gratefully acknowledged for providing the recorded SG data that were available in the GGP database.
This research is sponsored by the Polish Ministry of Science and Higher Education, grant No. 2011/01/B/ST10/05384

\section{REFERENCES}

Banka, D. and, Crossley, D.: 1999, Noise levels of superconducting gravimeters at seismic frequencies. Geophys. J. Int. 139, 87-97. DOI: 10.1046/j.1365-246X.1999.00913.x

Benedetto, J.J. and, Frazier, M.W.: 1994, Mathematics and Applications, LRC Press, Boca Raton, 221-245.

Bessissi, Z., Terbeche, M. and Ghezali, B.: 2009, Wavelet application to the time series analysis of DORIS station coordinates. C. R. Geoscience 341, 446-461. DOI: $10.1016 /$ j.crte.2009.03.010

Bogusz, J. and Figurski, M. : 2012, GPS-derived height changes in diurnal and sub-diurnal timescales. Acta Geophysica, 60, no. 2,

DOI: 10.2478/s11600-011-0074-5, 295-317.

Bogusz, J., Figurski, M., Kroszczyński, K. and Szafranek, K.: 2011, Investigation of environmental influences to the precise GNNS solutions. Acta Geodyn. Geomater., 8, No. 1 (161), 2011, 5-15.

Bogusz, J. and Hefty, J.: 2011, Determination of the notmodelled short periodic variations in the GPS permanent sites' positions. Acta Geodyn. Geomater., 8, No. 3 (163), 2011, 283-290.

Bogusz, J. and, Kontny, B. : 2011, Estimation of sub-diurnal noise level in GNSS time series. Acta Geodyn. Geomater., 8, No. 3 (163), 2011, 273-281.

Chamoli, A., Swaropa Rani, V., Srivastava, K., Srinagesh, D. and Dimri, V.P.: 2010, Wavelet analysis of the seismograms for tsunami warning. Nonlinear Processes in Geophysics, 17, Issue 5, 569-574. DOI: $10.5194 / \mathrm{npg}-17-569-2010$

Crossley, D. and, Hinderer, J.: 2010, GGP (Global Geodynamics Project): an international network of superconducting gravimeters to study time-variable 
gravity. IAG Symposia, Gravity, Geoid, and Earth Observation, 135, 627-635.

DOI: 10.1007/978-3-642-10634-7_83.

Crossley, D., Hinderer, J., Casula, G., Francis, O., Hsu, H.T., Imanishi, Y., Jentzsch, G., Kääriäinen, J., Merriam, J., Meurers, B., Neumeyer, J., Richter, B., Shibuya, K., Sato, T. and van Dam, T.: 1999, Network of superconducting gravimeters benefits a number of disciplines. EOS Transactions, 80, No. 11, 121-126.

Enescu, B., Ito, K. and Struzik, Z.: 2004, Wavelet-based multifractal analysis of real and simulated time series of earthquakes. Annuals of Disas. Prev. Res. Inst., Kyoto Univ., No. 47 B.

Greco, F., Budetta, G., Carbone, D., Panepinto, S. and Luzio, D.: 2007, The application of a denoising method aimed at reducing continuous gravity data. Communications to SIMAI Congress, 2. DOI: $10.1685 / C S C 06091$.

Gurley, K. and Kareem, A.: 1999, Application of wavelet transforms in earthquake, wind and ocean engineering. Engineering Structure 21, 149-167.

Hassler, S.K., Lark, R.M., Milne, A.E. and Elsenbeer, H.: 2011, Exploring the variation in soil saturated hydraulic conductivity under a tropical rainforest using the wavelet transform. European Journal of Soil Science, 62, 891-901. DOI: $10.1111 / \mathrm{j} .1365-2389.2011 .01400 . \mathrm{x}$

Hu, X.-G., Liu, L.-T., Ducarme, B., Xu, H.J. and Sun, H.-P.: 2007, Estimation of the pole tide gravimetric factor at the chandler period through wavelet filtering. Geophysical Journal International, 169 (3). DOI: 10.1111/j.1365-246X.2007.03330.x, 821-829.

Hu, X.-G., Liu, L.-T., Hinderer, J., Hsu, H.T. and Sun, H.P.: 2006, Wavelet filter analysis of atmospheric pressure effects in the long-period seismic mode band. Physics of the Earth and Planetary Interiors 154, 7084. DOI: 10.1016/j.pepi.2005.09.003

Hu, X.-G., Liu, L.-T., Hinderer, J. and Sun, H.-P.: 2005, Wavelet filter analysis of local atmospheric pressure effects on gravity variations. Journal of Geodynamics 79 (8), 447-459. DOI: $10.1007 / \mathrm{S} 00190-005-0486-6$

Kosek, W. and Popinski, W.: 2006, Forecasting of pole coordinates data by combination of the wavelet decomposition and autocovariance prediction. Proc. Journées 2005 Systèmes de Référence SpatioTemporels, 139-140.

Krause, P., Fink, M., Kroner, C. and Naujoks M.: 2009, The impact of hydrological processes on gravimetric measurements. J. Hydrology. 373(1-2), 151-163. DOI: $10.1016 /$ j.jhydrol.2009.04.019.

Kroner, C.: 2008, Report of activities of the IAG subcommission 3.1 special study group: "Analysis of environmental data for the interpretation of gravity measurements". Bull. d'Inf. Marées Terr. 144, 1154511546.
Kroner, C., Thomas, M., Dobslaw, H., Abe, M. and Weise, A.: 2009, Seasonal effects of non-tidal oceanic mass shifts in observations with superconducting gravimeters. New Challenges in Earth's Dynamics, Jena, September 1-5, 2008, J. Geodyn. 48(3-5), 354359. DOI: 10.1016/j.jog.2009.09.009.

Kroner, C. and Weise, A.: 2011, Sensitivity of superconducting gravimeters in central Europe on variations in regional river and drainage basins. J. Geod. 85(10), 651-659. DOI: $10.1007 / \mathrm{s} 00190-011-0471-1$.

Lark, R.M. and Webster, R.: 1999, Analysis and elucidation of soil variation using wavelets. European Journal of Soil Science, 50, 185-206.

Lintao, L., Houze, X., Heping, S. and Xinghua, H.: 2000, Wavelet approach to the determination of gravity tide parameters. Science in China (Series D). 43 No. 2, 158-165. DOI: 10.1007/BF02878145

Liye, Z. and Hongsheng, L: 2011, Adaptive morphological wavelet filter used in gravimeter signal processing. 2011 International Conference on Mechatronic Science, Electric Engineering and Computer. August 19-22. Jilin, China.

Lundstedt, H., Liszka, L., Lundin, R. and Muscheler, R.: 2006, Long-term solar activity explored with wavelet methods. Annales Geophysicae 24, 769-778.

Meyer, Y.: 1990, Ondelettes et Operateurs, 1, Hermann, Paris.

Misiti, M., Misiti, Y., Oppenheim, G. and Poggi, J.-M.: 2000, Wavelet toolbox. The MathWorks Inc.

Popinski, W.: 1996, Wavelet transform for time-frequency representation and filtration of discrete signals, Applicationes Mathematicae, 23, Nr. 4, WarszawaWrocław, 433-448.

Popinski, W. and Kosek, W.: 1995, Discrete fourier and wavelet transforms in analysis of Earth rotation parameters. Proc. Journees 1995 "Systemes de Reference Spatio-Temporels", Warsaw, Poland, Sep. $18-20,121-124$.

Sang, Y.F.: 2012, A practical guide to discrete wavelet decomposition of hydrologic time series. Water Resources Management 26, 3345-3365.

DOI: $10.1007 / \mathrm{s} 11269-012-0075-4$,

Singleton, R.C.: 1969, An algorithm for computing the mixed radix Fast Fourier Transform, IEEE Trans. Audio Electroacoust., v. AU-17, 93.

Xiaogang, H., Lintao, L., Heping, S., Houze, X., Hinderer, J. and Xiaoping, K.: 2006, Wavelet filter analysis of splitting and coupling of seismic normal modes below $1.5 \mathrm{mHz}$ with superconducting gravimeter records after December 26, 2004 Sumatra earthquake. Science in China Series D: Earth Sciences. 49, No. 12, 1259 1269. DOI: $10.1007 / \mathrm{s} 11430-006-1259-7$ 

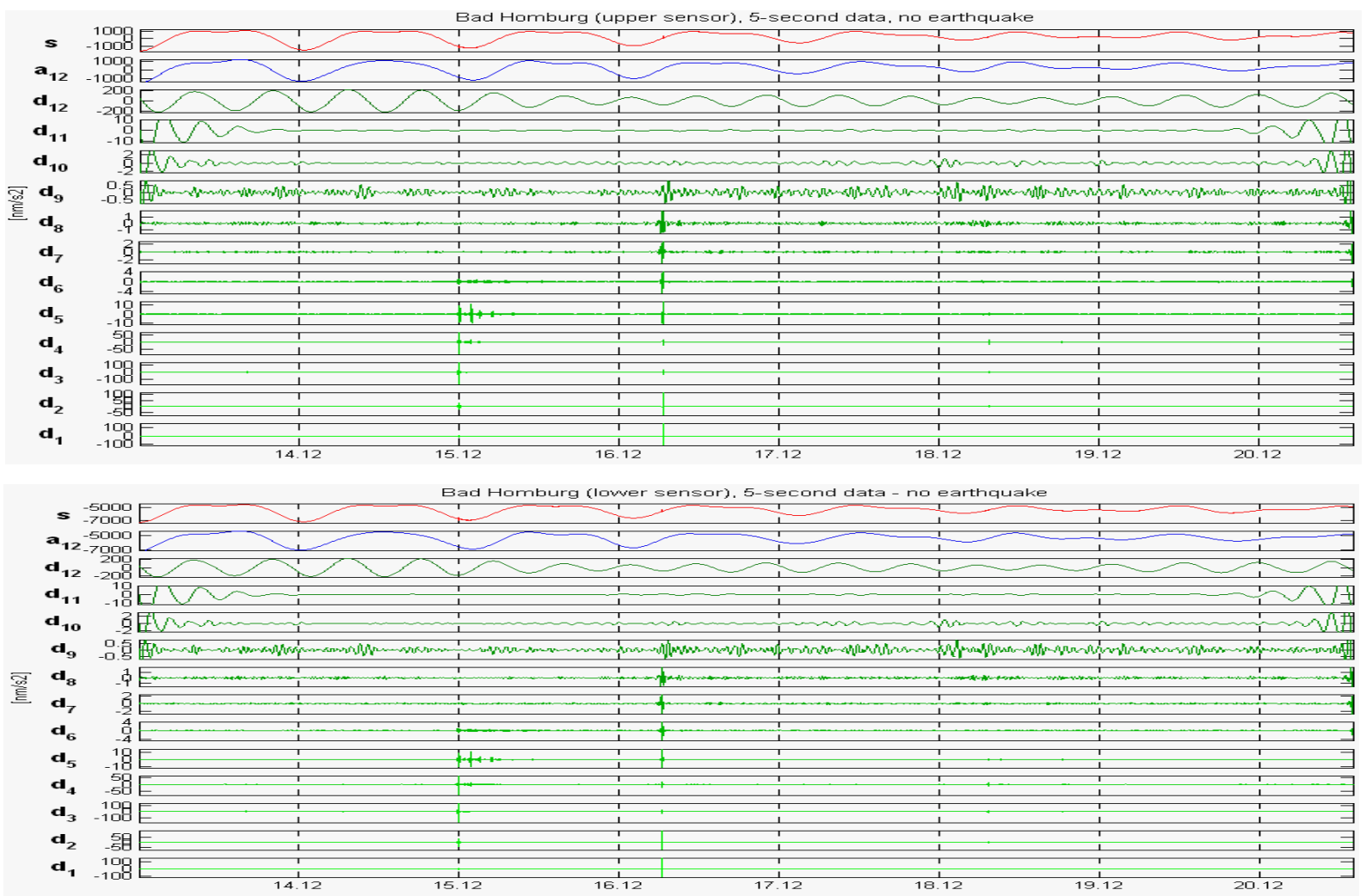

Fig. 5a Wavelet decomposition for the time series from the Bad Homburg station into $p=12$ frequency components during the quiet period; top: upper sensor, bottom: lower sensor.
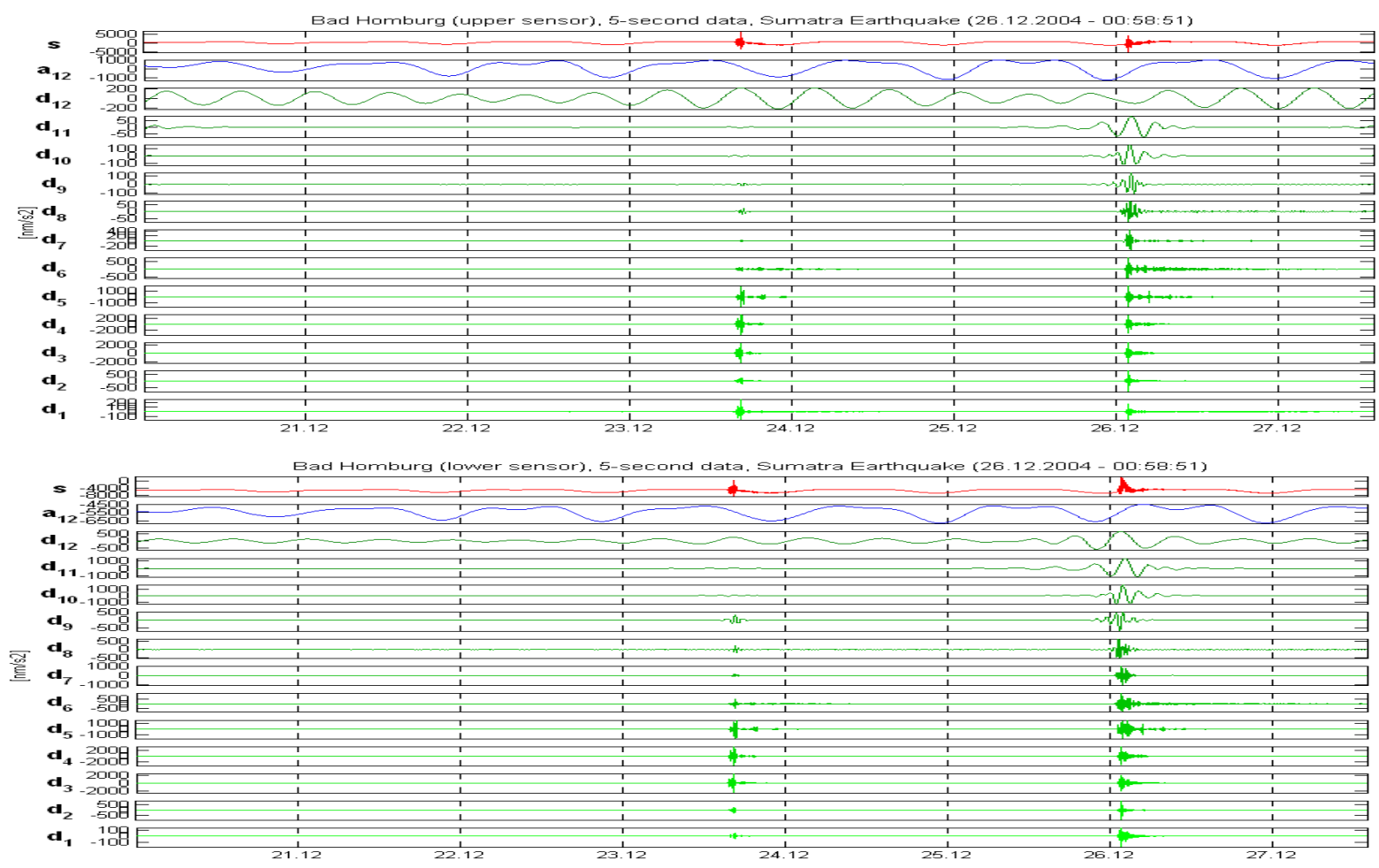

Fig. 5b Wavelet decomposition for the time series from the Bad Homburg station into $p=12$ frequency components during the Sumatra-Andaman earthquake; top: upper sensor, bottom: lower sensor. 



Fig. 6a Wavelet decomposition for the time series from the Wettzell station into $p=12$ frequency components during the quiet period; top: upper sensor, bottom: lower sensor.


Fig. 6b Wavelet decomposition for the time series from the Wettzell station into $p=12$ frequency components during the Sumatra-Andaman earthquake; top: upper sensor, bottom: lower sensor. 